\title{
DISTRIBUCIÓN ESPACIAL Y ANÁLISIS AMBIENTAL DE LAS PLANTAS RARAS DE LOS PIRINEOS
}

\section{Spatial distribution and environmental analysis of rare flora of the Pyrenees}

\author{
D. Gómez ${ }^{1 *}$, M. Lorda ${ }^{2,3}$, J. Garmendia ${ }^{3}$ y M.B. García ${ }^{1}$ \\ ${ }^{1}$ Instituto Pirenaico de Ecología (CSIC), 22700-Jaca (Huesca). \\ ${ }^{2}$ C.I. Agroforestal, 31015-Pamplona. \\ ${ }^{3}$ Sociedad de Ciencias Aranzadi, 20014-Donostia-San Sebastián \\ Identificador ORCID de los autores y e-mail: \\ D. Gómez: http://orcid.org/0000-0002-9738-8720 E-mail: dgomez@ipe.csic.es \\ M. Lorda: http://orcid.org/0000-0001-7335-3630 E-mail: mlordalo@educacion.navarra.es \\ J. Garmendia: http://orcid.org/0000-0001-5469-2207 E-mail: jgarmendia@aranzadi-zientziak.org \\ M.B. García: http://orcid.org/0000-0003-4231-6006 E-mail: mariab@ipe.csic.es \\ * Autor corresponsal
}

\begin{abstract}
Recibido: 19-04-2017. Aceptado: 17-08-2017. Fecha de publicación on-line: 20/10/2017
Citation / Cómo citar este artículo: Gómez, D., Lorda, M., Garmendia, J., García, M.B. (2017). Distribución espacial y análisis ambiental de las plantas raras de los Pirineos. Pirineos, 172, e030. doi: http://dx.doi.org/10.3989/ pirineos.2017.172005
\end{abstract}

RESUMEN: La reciente edición en línea del Atlas digital de Flora vascular de los Pirineos permite analizar algunos patrones de la distribución y las preferencias ambientales de las plantas más raras, con el fin de contribuir a la caracterización de la rareza y al diseño de estrategias de conservación de la diversidad florística en la cordillera. Entre los 363 taxones seleccionados por su escasa representación (el 10\% del total de la flora) 352 se distribuyen en menos del $2 \%$ del territorio y 11 ocupan un área inferior al $4 \%$, pero tienen menos de 30 de poblaciones en todo el territorio. La flora rara se distribuye en el $65 \%$ de los Pirineos (utilizando para esta estima una malla de cuadrículas UTM de $10 \mathrm{Km}$ de lado), y las regiones con más abundancia de plantas raras coinciden con las que tienen mayor diversidad florística total. Las zonas con mayor densidad de plantas raras se sitúan en el extremo oriental (junto al Cap de Creus), el noroccidental (entre San Sebastián e Irún) y en el centro del Parc National des Pyrénées y los alrededores de Monte Perdido. La flora rara se muestra significativamente diferente del conjunto de la flora en su representación taxonómica, distribución y amplitud altitudinal, corología, formas biológicas, preferencia edáfica y hábitats, pero no en el grado de naturalidad de los hábitats.

PALABRAS CLAVE: Distribución altitudinal; corología; formas biológicas; hábitats; naturalidad; rareza; Pirineos.

\begin{abstract}
The recent digital edition of the Atlas of Pyrenean vascular flora, allow us to analyze some patterns of geographical distribution and environmental features of the most rare plant species. The aim of our study is to contribute to the knowledge of rarity and to the planning and implementation of plant conservation at a Pyrenean scale. Among the 363 selected taxa after applying the criteria of low occupancy (10\% of the whole Pyrenean flora), 352 are present in less of the $2 \%$ of the territory and 11 occupy less than $4 \%$ distributed over a scarce number of populations
\end{abstract}


(less than 30). Using a net of $10 \times 10 \mathrm{Km}$ UTM's squares, rare plants appear represented in the $65 \%$ of the territory; the regions with highest rare plant density overlap those with the highest total plant number. The areas with the richest rare plants are placed at the East and West extremes (beside the Cap de Creus and at between San Sebastian and Irun respectively), at the center of the French National Park of the Pyrenees and the surroundings of the Spanish Monte Perdido area. Rare flora significantly differs from the whole flora in terms of taxonomic representation, altitudinal amplitude and distribution, chorology, life-forms, edaphic preference and habitats, but not in the naturalness of the habitats where they occur.

KEY WORDS: Altitudinal distribution; chorology; life-forms; habitats; habitat naturalness; rarity; Pyrenees.

\section{Introducción}

Las especies raras han atraído desde antiguo la atención de los naturalistas, y el estudio de su distribución y características biológicas ha sido muy relevante en el desarrollo de la biogeografía (Humboldt, 1805), la evolución (Darwin, 1859), la ecología (Griggs, 1940) y, ya recientemente, la filogeografía (Schönswetter \& Tribsch, 2005).

Las causas y consecuencias de la rareza han resultado muy atractivas en la ecología y se han interpretado a la luz de distintas hipótesis (Gaston, 1994) que, en muchos casos, restan todavía por elucidar (Fiedler \& Ahouse, 1992; Kunin \& Gaston, 1993 y 1997). Esta amplia utilización del concepto de rareza en la literatura biológica se ha basado en múltiples y a veces divergentes definiciones que han tenido en cuenta, entre otras variables, la abundancia, el área de distribución, la amplitud o especificidad de hábitat, la endemicidad, el aislamiento filogenético, junto a caracteres morfológicos, poblacionales y reproductivos (Rabinowitz, 1981; Kunin \& Gaston, 1993), lo que ha obligado a reflexionar sobre el propio significado del término (Gaston, 1997). Por otra parte, la rareza se muestra como una variable escala-dependiente en tiempo y espacio, lo que, dejando ahora a un lado la dimensión temporal, convierte el territorio de estudio en una cuestión fundamental para su análisis e interpretación y aconseja considerar distintas escalas del área de distribución (Rabinowitz, 1981). Por último, aunque se han descrito muchos métodos para estimar la abundancia de una especie determinada, sobre todo de fauna, el estudio de conjuntos de especies tan heterogéneos (en su tamaño, morfologías, tasas de crecimiento, modos de reproducción, etc.) como los que conforman las floras de territorios extensos resulta mucho más complejo (Blackburn \& Gaston, 1997).

En los Pirineos el estudio de las plantas raras se remonta al siglo XIX con la búsqueda de especies por describir (Bordère, 1866), de nuevos enclaves (Dulac, 1886) $\mathrm{y}$, ya en el siglo XX, con las prospecciones metódicas para la elaboración de numerosos catálogos florísticos en los que las plantas raras eran objeto de especial interés para interpretar y evaluar la flora y la vegetación. Más recientemente las plantas raras se han utilizado también en la detección de gradientes y en la comparación y delimitación ecológica de distintos territorios (Montserrat \& Montserrat, 1990), o en seguimientos demográficos para estimar su probabilidad de persistencia o extinción (García, 2003). Además, las plantas raras, junto a los endemismos y las situadas en su límite de distribución, han sido consideradas de forma prioritaria como objetivos de con- servación (Drury, 1974) y siguen siendo reseñadas en los catálogos y listas rojas de animales y plantas de muchos territorios (Villar et al., 1995; Carrascal \& Palomino, 2006; Lorda et al., 2009).

En la evaluación de las prioridades de conservación, las especies raras han atraído atención creciente en los últimos años en relación a su supervivencia, aunque quizás de forma excesiva, ya que, como se ha subrayado repetidamente, si bien el grado de rareza puede suponer un factor de riesgo, rareza y amenaza no son conceptos equivalentes (Gaston, 1994).

Pero sea cual sea el objetivo de su estudio y los métodos de análisis, la selección de plantas raras de un territorio requiere un registro preciso de su taxonomía y distribución, porque los sesgos derivados de los métodos utilizados para obtener dicha información pueden dar lugar a interpretaciones dispares en lo que atañe a la conservación de la diversidad biológica en sus distintas componentes - taxonómica, filogenética o funcional- (Pardo et al., 2017). Además, hay que considerar que el número y tamaño de las poblaciones constituyen indicadores parciales de la abundancia, tanto más cuando se vinculan a territorios delimitados por fronteras administrativas como sucede en la mayoría de listas rojas regionales y nacionales (Rodrigues \& Gaston, 2002).

En este marco, la edición digital de Atlas de la Flora Vascular de los Pirineos (http://www.florapyrenaea.org), con la revisión taxonómica y la recopilación y corrección de datos, permite afrontar el análisis de la flora de un amplio territorio que reúne un tercio de la flora europea en un amplio elenco ambiental, basándonos en un número relevante de registros florísticos y superando los sesgos impuestos por fronteras administrativas. En este trabajo delimitamos las plantas más raras de los Pirineos, su distribución territorial y altitudinal y la relación con algunas características básicas de los hábitats que ocupan, con el fin de contribuir a su caracterización biológica y ecológica y a la planificación de la conservación de la diversidad florística desde una perspectiva global de la cordillera.

\section{Material y métodos}

\subsection{Territorio de estudio}

El área de estudio abarca la cordillera de los Pirineos, con una superficie de unos $50.000 \mathrm{Km}^{2}$, un gradiente altitudinal de $3.400 \mathrm{~m}$ y una flora vascular de alrededor de 
3.650 taxones autóctonos. Los límites y características topográficas y ambientales del territorio quedan detallados en otro artículo de esta misma publicación (Gómez et al., 2017).

\subsection{Criterios taxonómicos y fuentes de información}

El criterio taxonómico y nomenclatural del Atlas de la Flora de Pirineos se expone de manera general en Gómez et al. (2017). La información utilizada en relación a las altitudes, pisos de vegetación, corología, preferencia edáfica, abundancia, hábitats y formas de vida, proceden de los datos de herbarios, inventarios y bibliográficos, tanto a nivel general (Flora iberica, Castroviejo et al., 1986-2015; Flora Gallica-Flore de France, Tison \& De Foucault, 2014), como de numerosos atlas, monografías y catálogos florísticos de territorios y especies de la cordillera pirenaica (Aizpuru et al., 1999; Dupias, 1985; Bolòs \& Vigo, 1984-2001; Villar et al., 1997-2001; Lorda, 2001, 2013).

\subsection{Selección de taxones para los análisis}

Los criterios más utilizados para la determinación del conjunto de especies raras son los de la amplitud del rango de la distribución y abundancia en un determinado territorio, o bien ambos aspectos (Rabinowitz, 1981). En nuestro caso, se ha seleccionando en primer lugar la amplitud de la distribución regional y luego, en las plantas en las que disponemos de esa información, la abundancia a escala territorial. Así, agrupamos las plantas más raras por la superficie total de ocupación en el área de estudio independientemente del número y densidad de sus poblaciones, más las que, aun ocupando una superficie algo mayor, tienen un número total de poblaciones muy reducido.

La información florística se ha basado en el Atlas de la Flora de los Pirineos antes citado, siguiendo su criterio nomenclatural y taxonómico. Se han considerando únicamente las plantas autóctonas con presencia reciente verificada en los Pirineos con los rangos taxonómicos de especie y subespecie. En los géneros apomícticos y otros de taxonomía compleja (Hieracium, Pilosella, Armeria, Taraxacum, Alchemilla, Festuca) se han excluido los taxones de difícil interpretación y los de área de distribución muy incierta. Además, se han excluido unos 20 taxones cuya rareza hemos juzgado como "accidental", ya que aunque mostrándose raros en el territorio (muchas veces por la escasa atención puesta en su prospección debido a su carácter banal, como sucede con muchas plantas ruderales) resultan muy abundantes en sus inmediaciones y ocupan además áreas muy extensas.

Con el objetivo de que nuestro trabajo sirva para propuestas de conservación de la biodiversidad, hemos considerado "plantas raras" las representadas entre 1 a 4 cuadrículas UTM de $10 \times 10 \mathrm{~km}$, es decir una superficie potencial de entre 100 y $400 \mathrm{~km}^{2}$, que supone entre el 0,2 y el $1 \%$ del territorio de los Pirineos. Además, hemos añadido un grupo de taxones que aun estando representados en más cuadrículas —entre 5 y $10 \mathrm{y}$, excepcionalmente hasta en 24- muestran un escasísimo número de poblaciones en el área de estudio (entre 1 y 3 por cuadrícula), faltan o son también muy raras en las regiones circundantes de la Península Ibérica y de Francia, y están recogidas en distintos catálogos de especies protegidas regionales y nacionales del entorno de los Pirineos. Aunque la información corológica disponible no permite conocer con detalle el número de poblaciones ni su tamaño para muchas plantas de la flora pirenaica, hemos recurrido a cifras aproximadas extraídas de otras floras (Gómez et al., 2005) u obtenidas mediante "criterio de experto"; las áreas globales de distribución se han extraído de Flora iberica, Castroviejo et al., 1986-2015, Flora europaea (Tutin et al., 1964-1980) y de los mapas de distribución de GBIF, http://www.gbif.org.

\subsection{Análisis de datos}

Para determinar si la distribución de la flora rara de los Pirineos en los distintos criterios bióticos y abióticos considerados es diferente del conjunto de la flora (excluidas las plantas del estudio), hemos empleado el test de Chi-quadrado. La Tabla 1 resume los criterios y las clases consideradas.

\section{Resultados}

\subsection{Número de taxones raros}

Con los criterios de selección señalados se obtuvieron un total de 363 plantas raras, lo que supone el 10\% del total de la flora vascular pirenaica. La amplitud de su distribución en los Pirineos, por el número de cuadrículas de 10x10 $\mathrm{Km}^{2}$, es la siguiente: 57 taxones (el 15,7\% del conjunto de las raras) están presentes en una cuadrícula, 70 (el 19,3\%) en dos cuadrículas, 68 (el 18,7\%) en tres cuadrículas, 52 (14,3\%) en cuatro cuadrículas, 104 (el 28,6\%) entre 5 y 10 cuadrículas y 12 (el 3,3\%) entre 10 y 24 cuadrículas, este último grupo con un número total de poblaciones menor de 30. El listado completo de plantas de cada uno de estos grupos puede consultarse en Gómez et al. (2016).

En esta selección están representados algunos taxones que en territorios próximos muestran mayor abundancia por encontrarse vinculados a hábitats particulares, muy localizados en general y con escasa presencia en el Pirineo - como los suelos salinos o yesosos-, o bien son irradiaciones extremas de la flora mediterránea —o atlántica en unos pocos casos - que alcanza la zona basal de los Pirineos. Es el caso de Helianthemum squamatum (L.) Pers., H. origanifolium (Lam.) Pers. subsp. origanifolium, Jacobaea auricula (Bourgeau ex Coss.) Pelser, Narcissus triandrus L. subsp. triandrus y subsp. pallidulus (Graells) Rivas Goday, Urginea maritima (L.) Baker, Herniaria ciliolata Melderis, Leucanthemum crassifolium (Lange) Lange, Romulea bulbocodium (L.) Sebast. \& Mauri., Anthyllis cytisoides L., Cistus psilosepalus Sweet, Cytisus 
Tabla 1: Resumen de los factores analizados mediante test de Chi-cuadrado para comparar la flora rara con la del conjunto de los Pirineos, excluidas las raras. Se detalla el número de clases y su descripción.

Table 1: Summary of factors for which differences between the Pyrenean endemisms and the entire flora (excluding the rare plants) where tested by a Chi-square test. Number of classes and their description is detailed.

\begin{tabular}{|l|c|l|}
\hline \multicolumn{1}{|c|}{ Factor testado } & $\mathbf{N}^{\mathbf{0}}$ Clases & \multicolumn{1}{c|}{ Descripción de las clases } \\
\hline Taxonomía (grandes grupos) & 4 & Pteridofitos, Gimnospermas, Dicotiledóneas, Monocotiledóneas. \\
\hline Amplitud geográfica & 6 & Especies presentes en 1,2,3,4,5 o 6 sectores geográficos de los Pirineos \\
\hline Corología & 6 & $\begin{array}{l}\text { Amplia distribución, alpinas, boreo-alpinas, endémicas, eurosiberianas, } \\
\text { mediterráneas. }\end{array}$ \\
\hline Pisos de vegetación & 7 & $\begin{array}{l}\text { Basal mediterráneo; basal atlántico; submontano; montano; subalpino; alpino; } \\
\text { subnival. }\end{array}$ \\
\hline Amplitud altitudinal & 6 & $\begin{array}{l}\text { Entre 0-499m; 500-999m; 1.000-1.499m; 1.500-1.999m; 2.000-2.499m; 2.500- } \\
2.999 m . ~\end{array}$ \\
\hline Hábitat & 6 & $\begin{array}{l}\text { Costas y suelos salobres; bosques; matorrales; roquedos y gleras; humedales; } \\
\text { pastos. }\end{array}$ \\
\hline Naturalidad del hábitat & 4 & Muy alta, alta, intermedia, baja. \\
\hline Forma biológica & 8 & $\begin{array}{l}\text { Epifitos; hidrófitos; macrofanerófitos; nanofanerófitos; geófitos; caméfitos; } \\
\text { terófitos; hemicriptófitos. }\end{array}$ \\
\hline Afinidad edáfica & 3 & Indiferente; acidófilo; basófilo. \\
\hline
\end{tabular}

commutatus (Willk.) Briq., Erodium daucoides Boiss., Launaea pumila (Cav.) Kuntze, Onopordum corymbosum Willk., O. nervosum Boiss., Picris hispanica (Willd.) P.D. Sell, Thymus mastichina L. subsp. mastichina, Th. zygis L. subsp. zygis, Coleostephus myconis (L.) Rchb. f., Klasea flavescens (L.) Holub subsp. leucantha (Cav.) Cantó \& Rivas Mart., Marrubium supinum L., Senecio carpetanus Boiss. \& Reuter, Stipa lagascae Roem. \& Schult., Teucrium gnaphalodes L'Hér., Atractylis cancellata L. y Sideritis montana L. subsp. montana.

Entre las plantas que hemos incluido con área de ocupación más amplia, pero muy escasas poblaciones, hay que destacar las orquídeas Corallorhiza trifida Chatel., Cypripedium calceolus L. y Epipogium aphyllum Swartz. y los taxones de óptimo atlántico, limitados al País Vasco y Navarra: Prunus lusitanica L. subsp. lusitanica, Senecio nemorensis L. subsp. bayonnensis (Boiss.) Nyman, y el helecho de afinidad atlántico-macaronésica Vandenboschia speciosa (Willd.) Kunkel.

\subsection{Distribución taxonómica}

De los grandes grupos taxonómicos, encontramos entre las plantas raras la siguiente representación: PTERIDOPHYTA (helechos, isoetes y licopodios), 18 taxones (el 5\% del total); ANGIOSPERMAE Dicotiledóneas 260 taxones (el 71,8\%) y ANGIOSPERMAE Monocotiledóneas 85 taxones (el 23,4\%), no encontrándose ninguna gimnosperma en la selección. En conjunto, la frecuencia de especies de flora rara dentro de los grupos de helechos, gimnospermas, monocotiledóneas y dicotiledóneas difiere significativamente del conjunto de la flora (Tabla 3), siendo más abundantes de lo esperado los helechos y monocotiledóneas y menos los demás grupos.
De las 145 familias que forman la flora del Pirineo, 82 $(56,6 \%)$ están representadas en la flora rara. Destacan por su número las familias Asteráceas $(9,6 \%$ de las plantas raras), Fabáceas $(7,4 \%)$, Cariofiláceas $(7,1 \%)$, Ciperáceas (6\%) y Poáceas $(5,5 \%)$. El resto - 77 familias - engloban menos del 5\% de las plantas raras. En comparación con el conjunto de la flora pirenaica, las plantas raras aparecen sobrerrepresentadas en las familias Boragináceas, Cariofiláceas, Lamiáceas y Ciperáceas; por el contrario, en las Asteráceas, Rosáceas, Brasicáceas y Escrofulariáceas las plantas raras muestran frecuencias de la mitad o menos que en el total. En conjunto, la flora rara muestra una distribución taxonómica significativamente diferente de la del conjunto de la flora pirenaica (Figura 1).

\subsection{Distribución territorial}

Las plantas raras se distribuyen en los Pirineos de forma muy heterogénea (Figura 2), sobresaliendo notablemente por la mayor concentración en el extremo oriental, junto al Cap de Creus. Un segundo núcleo, mucho menos destacado está en la vertiente nororiental, centrado entre el Puigmal y el Macizo del Canigó, en el Parque regional de los Pirineos catalanes. También resalta el extremo noroccidental, entre San Sebastián e Irún y el Parque natural Aiako harria. Por último, el sector central del Parc National des Pyrénées, en la zona de Gavarnie, y Néouvielle en la vertiente francesa y la zona vecina del Macizo de Monte Perdido en la vertiente española. En total, las plantas raras están representadas en 358 cuadrículas (el 65\% del total), pero solo 10 cuadrículas albergan más de 25 taxones raros.

La distribución por regiones administrativas de la cordillera (Tabla 2) muestra la mayor representación en Ca- 


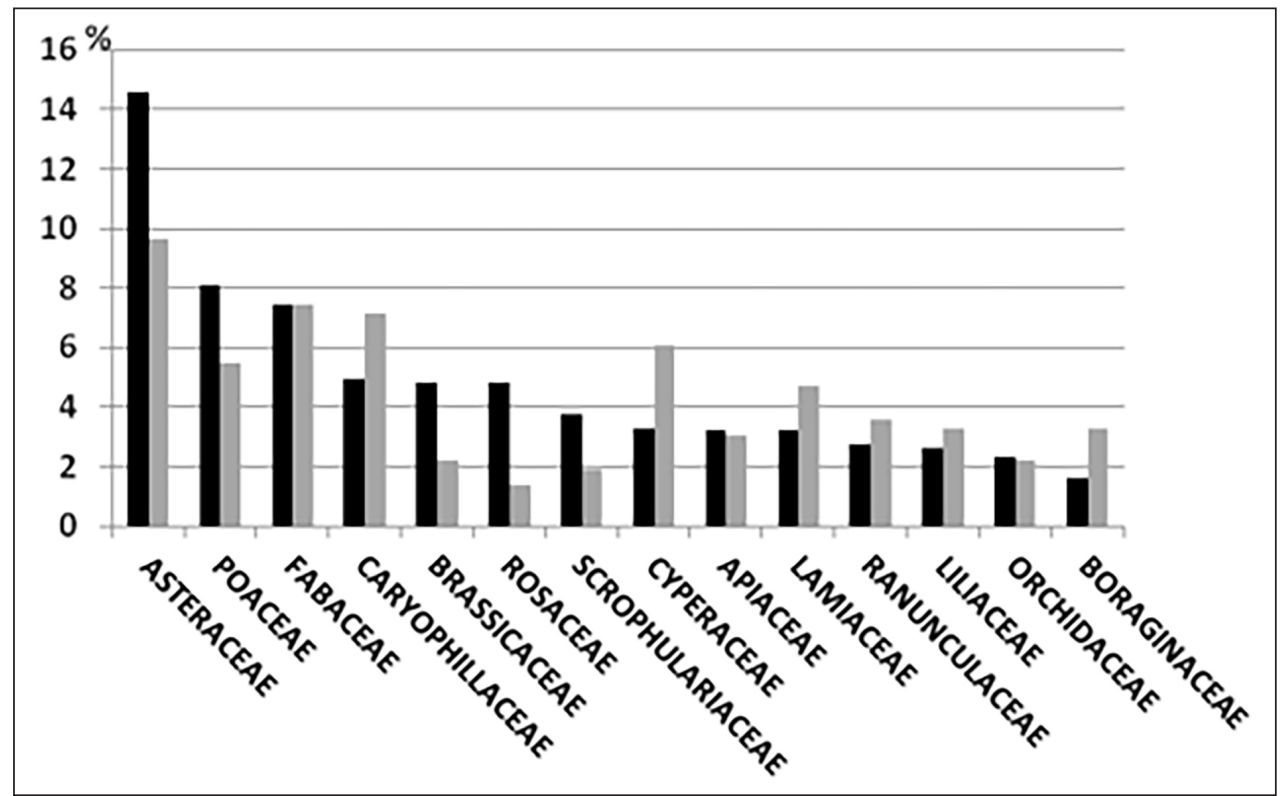

Figura 1: Distribución de familias taxonómicas frecuentes entre la flora "rara" (columnas en gris) y del conjunto de la flora de los Pirineos (columnas en negro).

Figure 1: Taxonomic families distribution among the Pyrenean rare plants (grey columns) and the total Pyrenean flora (black columns).

taluña, que alberga más de la mitad de la flora rara, seguida de Pyrénées Orientales, Aragón, Navarra y el País Vasco. En el otro extremo, Andorra muestra un escaso número de taxones raros (3\%). Sin embargo, ponderando por la superficie de cada región, destaca notablemente la cifra de los Pyrénées Orientales.

Respecto a los sectores geográficos (Figura 3), el Oriental norte es el más rico en flora rara (158 taxones), seguido del Central sur (121) y del Occidental sur (110). El sector más pobre es el Occidental norte (36 taxones). La representación de plantas raras en los distintos sectores muestra una variación notablemente mayor (entre el 3 y el $52,3 \%$ ) que la del conjunto de la flora que está representada en todos los sectores con entre el 50 y el $75 \%$ de taxones.

\subsection{Distribución y amplitud altitudinal}

Respecto al gradiente altitudinal de los Pirineos, la mayor presencia de taxones raros se localiza por debajo

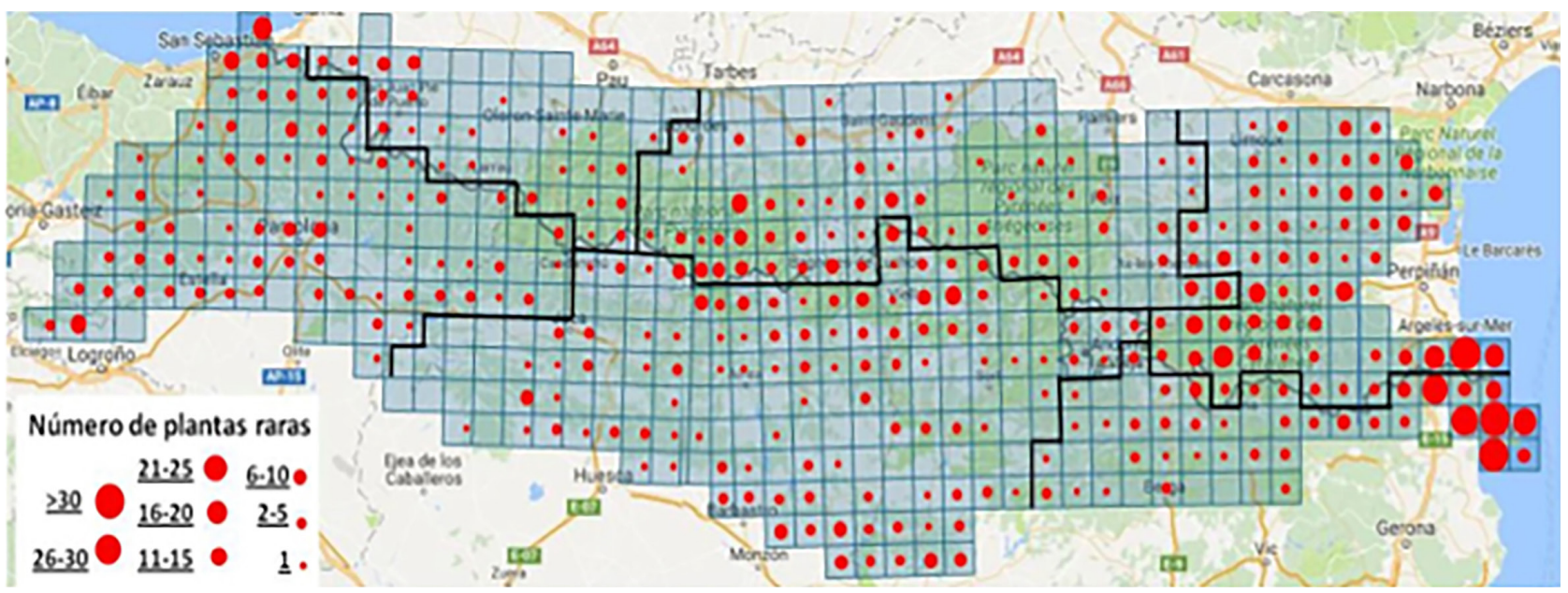

Figura 2: Mapa de distribución de la flora rara en los Pirineos. El tamaño de los puntos representa el número de plantas raras presentes en cada cuadrícula de 10 x $10 \mathrm{Km}$.

Figure 2: Pyrenees distribution map of the rare plants. Dot sizes represent the number of rare plants occurring in each $10 \times 10 \mathrm{Km}$ square. 
Tabla 2: Número de plantas raras y del total de la flora en las 11 regiones administrativas de los Pirineos. Table 2: Number of rare plants and total flora in the 11 administrative regions of the Pyrenees.

\begin{tabular}{|l|c|c|c|c|c|}
\hline \multicolumn{1}{|c|}{ Región/Departamento } & $\begin{array}{c}\mathbf{N}^{\mathbf{0}} \text { taxones } \\
\text { raros }\end{array}$ & $\begin{array}{c}\text { \% del total } \\
\text { raras }\end{array}$ & $\begin{array}{c}\mathbf{N}^{\mathbf{0}} \text { taxones } \\
\text { totales }\end{array}$ & $\begin{array}{c}\text { Superficie } \\
\mathbf{K m}^{\mathbf{2}}\end{array}$ & $\begin{array}{c}\mathbf{N}^{\mathbf{0}} \text { taxones raros/100 Km } \\
\text { de superficie }\end{array}$ \\
\hline Andorra & 11 & 3 & 1393 & 452 & 2,4 \\
\hline Haute-Garonne & 21 & 5,8 & 1653 & 1420 & 1,4 \\
\hline Ariège & 31 & 8,5 & 1774 & 3993 & 0,7 \\
\hline Pyrénées Atlantiques & 32 & 8,8 & 1790 & 3697 & 0,8 \\
\hline Hautes-Pyrénées & 50 & 13,8 & 1848 & 3104 & 1,6 \\
\hline Aude & 58 & 16 & 2057 & 2648 & 2,2 \\
\hline País Vasco & 62 & 17,1 & 1848 & 2772 & 2,2 \\
\hline Navarra & 76 & 20,9 & 2339 & 6658 & 1,1 \\
\hline Aragón & 99 & 27,3 & 2591 & 10803 & 0,9 \\
\hline Pyrénées Orientales & 135 & 37,2 & 2529 & 3226 & 4,2 \\
\hline Cataluña & 190 & 52,3 & 2958 & 12264 & 1,5 \\
\hline
\end{tabular}

de los $1.000 \mathrm{~m}$ de altitud (286 taxones, $79 \%$ del total), decreciendo notablemente en alturas más elevadas: 59 taxones entre 1.000 y $1.500 \mathrm{~m}, 42$ entre 1.500 y $2.000 \mathrm{~m}$, 23 entre 2.000 y $2500 \mathrm{~m}$, y únicamente 3 taxones sobrepasan los $2500 \mathrm{~m}$ de altitud (Figura 4).

Por pisos de vegetación, el $63,2 \%$ de la flora rara se encuentra en el piso basal (frente al $61,2 \%$ del conjunto de la flora); el $48,2 \%$ en el montano inferior (frente al $71,6 \%$ ), el $35,6 \%$ en el montano superior (frente al $73,2 \%$ ), el $16,8 \%$ en el subalpino (frente al $38,1 \%$ ), el $8,1 \%$ en el alpino (frente al $17 \%$ ) y el $1,2 \%$ en el nival, sobre $2800 \mathrm{~m}$, (frente al 4,8\%).

Considerando la amplitud altitudinal que muestran las plantas raras (Figura 5), el 56\% muestra un rango menor de $500 \mathrm{~m}$, el $32 \%$ entre 500 y $1.000 \mathrm{~m}$ y apenas un $12 \%$ supera dicha amplitud. El contraste con el conjunto de la flora es muy notable y muestra diferencias significativas (Tabla 3).

\subsection{Corología}

La distribución corológica de las plantas raras (Figura 6), en comparación con la flora general, muestra una mayor representación de las mediterráneas $(42,8 \%$ frente al $31,6 \%$ en el conjunto de la flora) y las boreoalpinas (5,5\% frente al 3,6\%) y una menor representación de las eurosiberianas $(27,5 \%$ frente al $34,9 \%)$, las de "amplia distribución" (10,4\% frente al 14,1\%) y los orófitos alpinos $(8 \%$ frente al $10,3 \%)$. Los endemismos pirenaicos tienen una proporción similar $(5,8 \%$ y $5,3 \%)$. En conjunto,

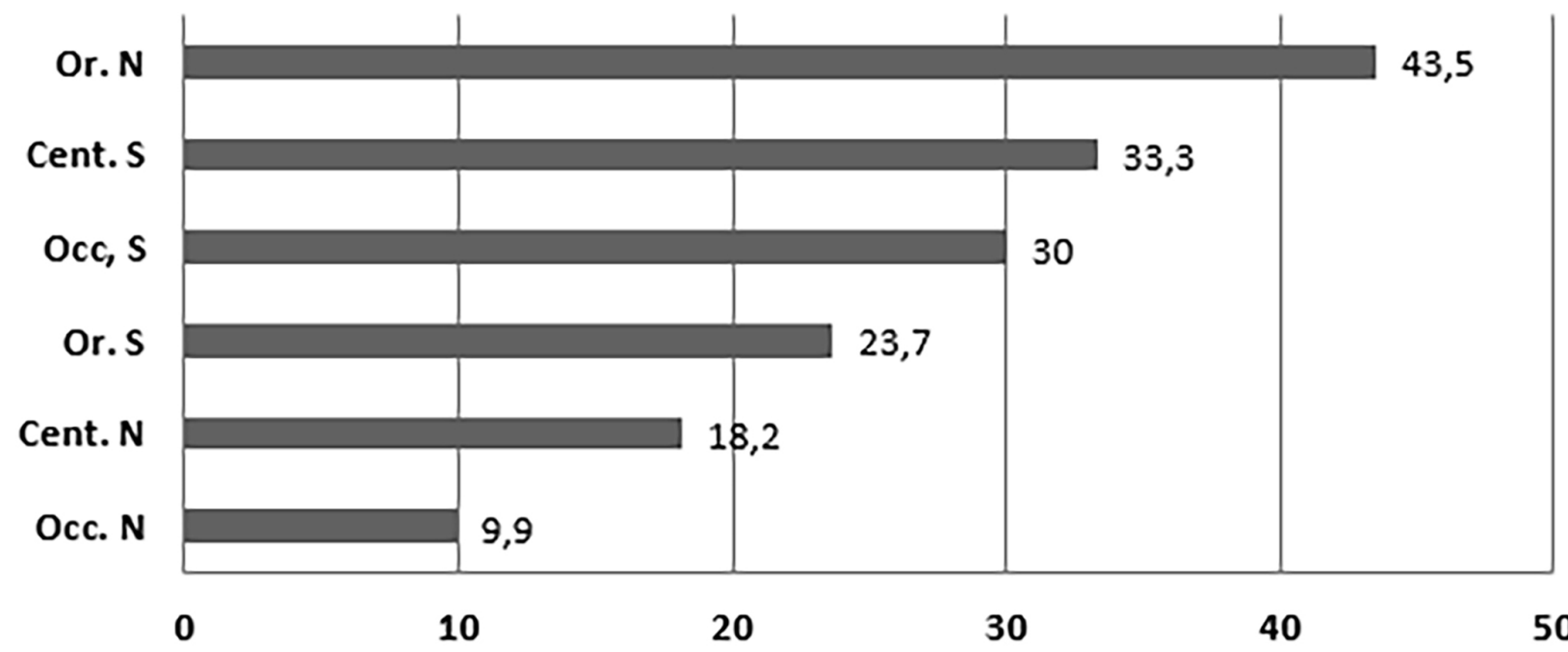

Figura 3: Porcentaje de las plantas raras (respecto al total en cada sector) en los sectores geográficos de los Pirineos. Figure 3: Percentage of rare plants (with respect to the total in each sector) in the geographic sectors of the Pyrenees. 


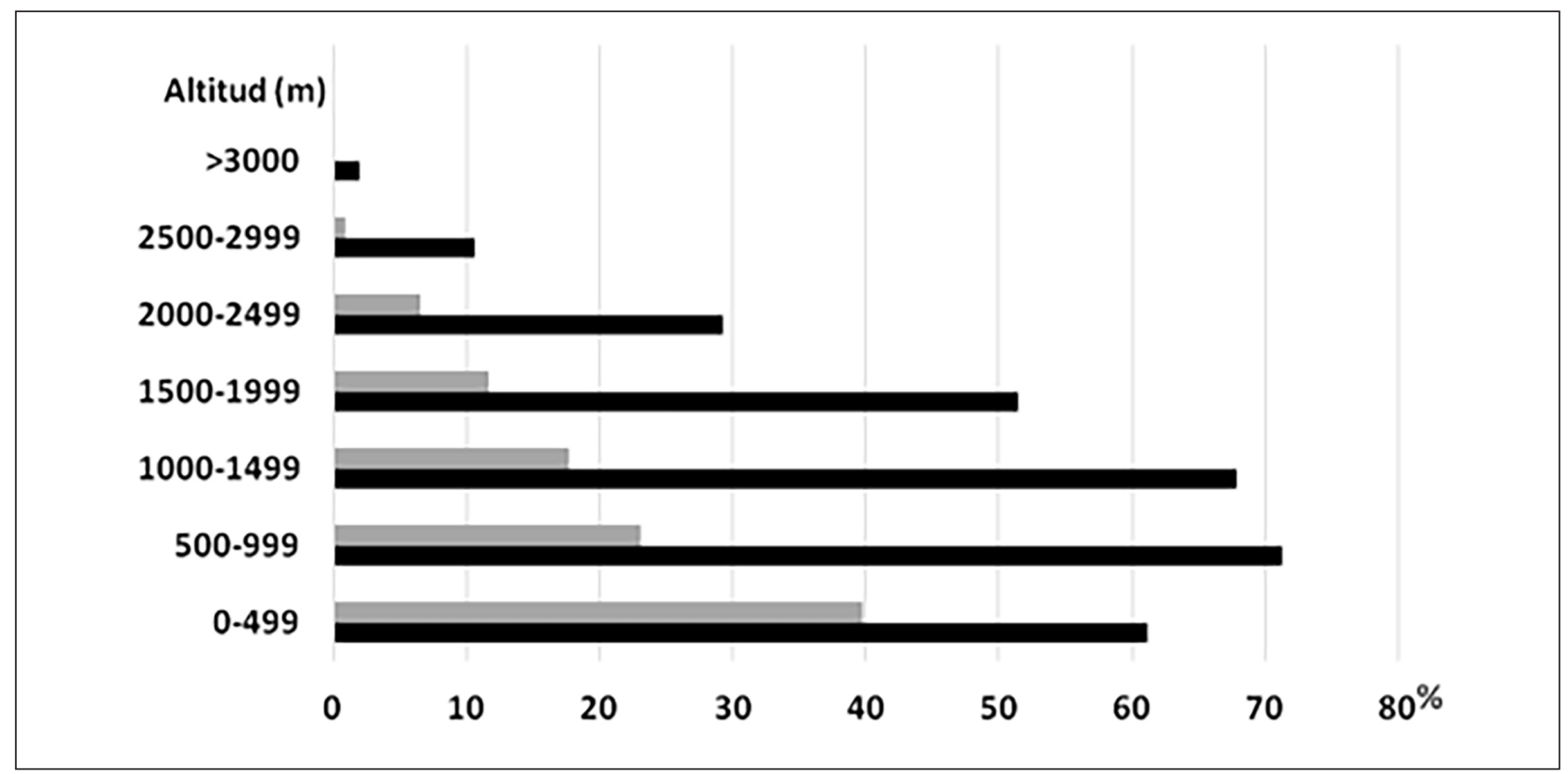

Figura 4: Porcentaje de las plantas raras (columnas grises) y del total de la flora (columnas negras) en el gradiente altitudinal de los Pirineos.

Figure 4: Percentage of rare plants (grey columns) and of the total flora (black columns) along the altitudinal gradient of the Pyrenees.

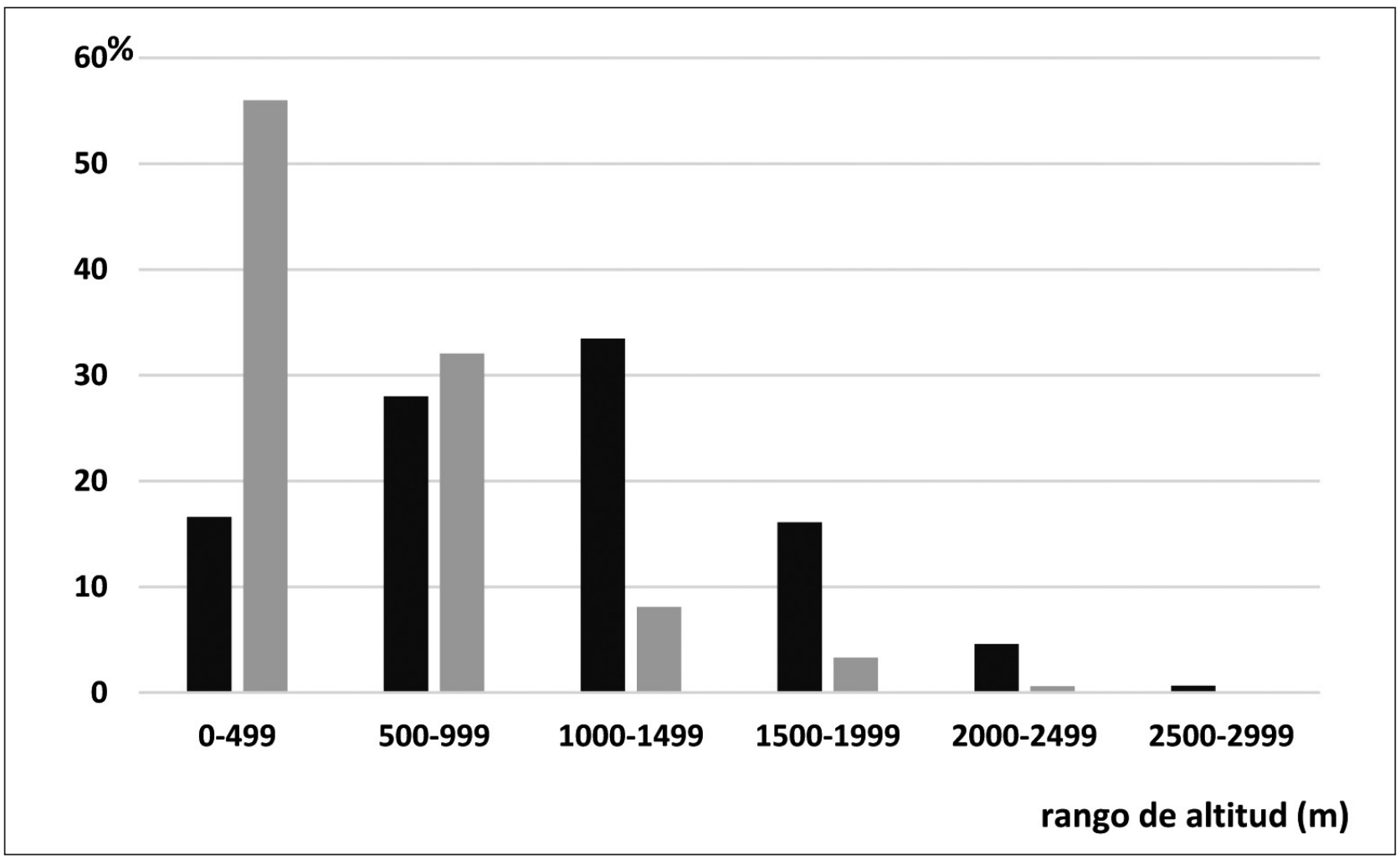

Figura 5: Amplitud altitudinal —en intervalos de $500 \mathrm{~m}$ - de las plantas raras (columnas grises) y el conjunto de la flora (columnas negras) de los Pirineos.

Figure 5: Altitudinal range (intervals of $500 \mathrm{~m}$ ) of rare plants (grey columns) and the total flora (black columns) of the Pyrenees. 
Tabla 3: Resumen de los test de Chi-cuadrado detallando la hipótesis nula, los grados de libertad, la significación del valor de P (NS: no significativo, $* * *: \mathrm{P}<0,001)$ y una breve interpretación del resultado.

Table 3: List of the 7 Chi-square tests performed. The null Hypothesis is listed, with the degrees of freedom, the significance of the $P$-value of the test (NS no significant, ***: $p$-value $<0,001)$ and a short interpretation which is detailed in the text.

\begin{tabular}{|l|c|c|l|}
\hline \multicolumn{1}{|c|}{ Test (H0:) } & df & p-valor & \multicolumn{1}{|c|}{ Interpretación } \\
\hline $\begin{array}{l}\text { La distribución taxonómica de la flora rara es } \\
\text { similar a la del conjunto de la flora (excluidas } \\
\text { las plantas raras). }\end{array}$ & 3 & $* * *$ & $\begin{array}{l}\text { Los grupos taxonómicos de las plantas raras del Pirineo son } \\
\text { diferentes de los del conjunto de la flora. }\end{array}$ \\
\hline $\begin{array}{l}\text { La corología de la flora rara es similar a la del } \\
\text { conjunto de la flora. }\end{array}$ & 5 & $* * *$ & $\begin{array}{l}\text { Las plantas raras tienen un espectro corológico distinto del } \\
\text { conjunto de la flora. }\end{array}$ \\
\hline $\begin{array}{l}\text { La amplitud altitudinal de la flora rara es } \\
\text { similar a la del conjunto de la flora. }\end{array}$ & 5 & $* * *$ & $\begin{array}{l}\text { Las plantas raras tienen una menor amplitud altitudinal que } \\
\text { el conjunto de la flora. }\end{array}$ \\
\hline $\begin{array}{l}\text { La distribución por hábitats de la flora rara es } \\
\text { similar a la del conjunto de la flora. }\end{array}$ & 6 & $* * *$ & $\begin{array}{l}\text { Las plantas raras de los Pirineos tienen una distribución por } \\
\text { hábitats diferente del conjunto de la flora. }\end{array}$ \\
\hline $\begin{array}{l}\text { La naturalidad de los hábitats de la flora rara es } \\
\text { similar a la del conjunto de la flora. }\end{array}$ & 3 & ns & $\begin{array}{l}\text { No se puede afirmar que las plantas raras de los Pirineos } \\
\text { vivan en hábitats con distinta naturalidad que el conjunto de } \\
\text { la flora. }\end{array}$ \\
\hline $\begin{array}{l}\text { La distribución de formas biológicas de la flora } \\
\text { rara es similar a la del conjunto de la flora. }\end{array}$ & 7 & $* * *$ & $\begin{array}{l}\text { Los terófitos e hidrófitos están sobrerrepresentados entre la } \\
\text { flora rara de los Pirineos en relación al conjunto de la flora. }\end{array}$ \\
\hline $\begin{array}{l}\text { La afinidad edáfica de la flora rara es similar a } \\
\text { la del conjunto de la flora. }\end{array}$ & 2 & $* * *$ & $\begin{array}{l}\text { Las plantas raras de los Pirineos tienen una afinidad edáfica } \\
\text { distinta de la del conjunto de la flora. }\end{array}$ \\
\hline
\end{tabular}

la corología de la flora rara es significativamente diferente de la del total de los Pirineos (Tabla 3).

Del total de la flora rara solo 19 taxones se consideran endémicos, 14 a nivel de especie y 5 de subespecie. Por tanto, del total de endemismos pirenaicos, 128 taxones, sólo se pueden considerar raros el 14,8\% según los criterios que hemos considerado en la definición de "rareza".

175 plantas raras (el 48,5\% del total) encuentran en el Pirineo su límite de distribución. De ellas, 96 muestran su límite N, NE o NW en los Pirineos; este grupo está formado mayoritariamente (cerca del $90 \%$ ) por taxones de distribución mediterránea que no traspasan la vertiente meridional de los Pirineos. En sentido opuesto, 74 plantas raras tienen en el Pirineo su límite meridional ( $\mathrm{S}$ o SW), agrupando principalmente taxones eurosiberianos $(40,5 \%)$, orófitos alpinos $(29,7 \%)$ y boreo-alpinos $(22,9 \%)$. Por último, 5 plantas atlánticas alcanzan su límite oriental en la cordillera.

\subsection{Espectro de formas biológicas}

La distribución de las formas biológicas (Figura 7) está dominada por los hemicriptófitos $(29,7 \%)$ y los terófitos $(28,4 \%)$. Estos últimos y los hidrófitos se muestran notablemente más representados entre las plantas raras que en el conjunto de la flora, al igual que los caméfitos y los geófitos que destacan en menor proporción. Por el contrario, hemicriptófitos, fanerófitos y epífitos aparecen subrepresentados entre las plantas raras. En conjunto, la distribución de formas biológicas entre las plantas raras es significativamente diferente de la del conjunto de la flora (Tabla 3).

\subsection{Preferencia edáfica}

Las plantas acidófilas suponen un 27,8\% de los taxones raros (frente al 21,4\% del conjunto de la flora), mientras que las basófilas y las indiferentes al substrato muestran valores similares, próximos al 36\% (34,8 y 43,4\% respectivamente en el conjunto de la flora). En conjunto, las plantas raras se muestran distintas significativamente en su afinidad edáfica (Tabla 3). Entre la flora rara se encuentran 7 taxones gipsófitos y 13 halófitos que señalan la presencia muy localizada de dichos substratos en la parte basal de los Pirineos.

\subsection{Distribución por hábitats y naturalidad}

Atendiendo a su hábitat preferencial, las plantas raras se localizan principalmente en los pastos y humedales (Figura 8). Respecto al conjunto de la flora, están más representadas en los suelos salobres de la costa y del interior y en los humedales, menos representadas en los pastos, bosques y matorrales, y muestran una representación similar en el resto de hábitats. En comparación con el conjunto de la flora, la distribución por hábitats de las plantas raras es significativamente diferente (Tabla 3).

Respecto al grado de naturalidad de los hábitats, 185 taxones (51\% flora rara) viven en hábitats de alta o muy alta naturalidad destacando los humedales y los roquedos, seguidos de las comunidades forestales, los pastos, las costas y los medios salinos y, finalmente, los matorrales. Una naturalidad media acoge 99 taxones, y una baja naturalidad, coincidente con los ambientes más intervenidos pastos degradados, matorrales, zonas de cultivos-, incluye 74 taxones. En conjunto, la distribución de las plantas 


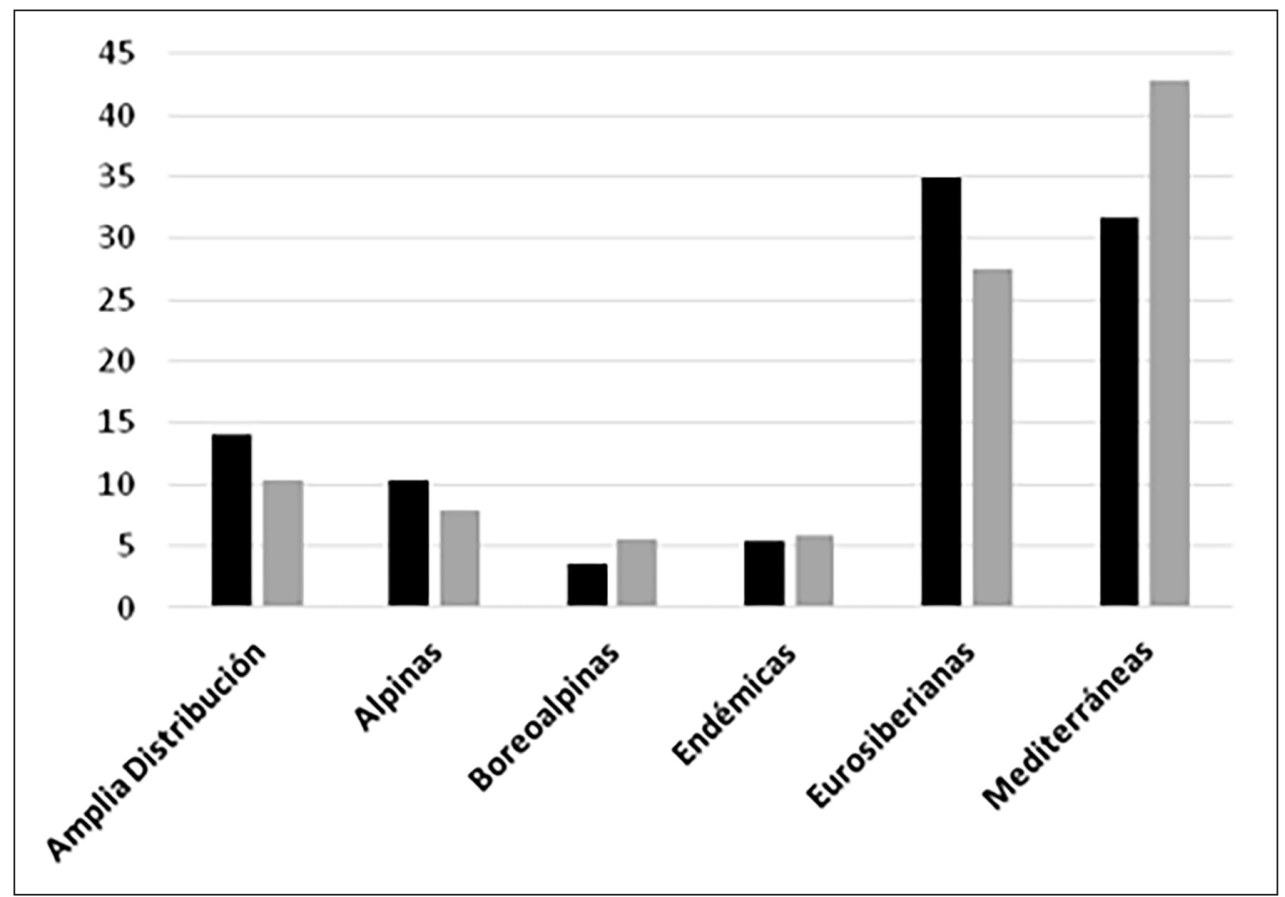

Figura 6: Espectro corológico de las plantas raras (columnas grises) y del conjunto de la flora (columnas negras) de los Pirineos.

Figure 6: Chorology spectrum of the rare plants (grey columns) and the total flora (black columns) of the Pyrenees.

raras por la naturalidad del hábitat que ocupan no es significativamente diferente del conjunto de la flora (Tabla 3).

\section{Discusión y conclusiones}

Las distintas perspectivas con que se ha interpretado el concepto de rareza en diferentes estudios y con múltiples objetivos, han dificultado la adopción de criterios no subjetivos y normalizados para la delimitación de las plantas raras (Carroll et al., 1996; Blackburn \& Gaston, 1997; Keith, 1998). Aunque la presencia de especies, medida por el número de celdas ocupadas en un área de estudio ("species occupancy"), se ha usado con más frecuencia y se considera un buen indicador de la abundancia (He $\&$ Condit, 2007), hay que señalar las limitaciones y sesgos irremediables que pueden derivarse de la escasez e imprecisión de los datos disponibles (Batianoff \& Burgess, 1993; Domínguez et al., 2003). En los Pirineos, aun contando con un número muy destacable de citas florísticas (cerca de dos millones), todavía es escasa la prospección en algunas zonas y existen muchas lagunas en el conocimiento taxonómico de determinados grupos florísticos y en el número y densidad de las poblaciones de no pocas plantas. Con estas limitaciones, las 363 plantas seleccionadas (algo menos del 10\% del total de la flora), constituyen una buena base para indagar las prioridades de conservación (Domínguez et al., 2003). Alrededor de 130 de esas plantas (el 36\%) figuran ya en alguno de los "Catálogos regionales".
La ausencia de estudios similares en otros territorios montañosos nos impide comparar las cifras que hemos seleccionado como flora rara. Si nos fijamos únicamente en la distribución en la cordillera, y dejando a un lado el número de poblaciones, 247 plantas (un 6,7\% del total) ocupan menos del 1\% del territorio. Sin embargo, cabe destacar que el número de plantas de los Pirineos que ocupa una superficie reducida y podría considerarse rara es muy elevado, pues cerca de la mitad de la flora está presente en menos del $6 \%$ de la cordillera. Por el contrario, menos del 15\% del total de las plantas está representada en más del 30\% del territorio (Gómez et al., 2017). Estas cifras señalan la preponderancia en los Pirineos de las plantas "raras" sobre las "comunes", como se ha encontrado ya en muchos otros territorios (Gaston, 1994).

Respecto a su distribución espacial, hay que destacar el predominio de plantas raras en las zonas situadas a menor altitud y, en parte, en la vecindad de áreas con alta densidad humana, lo que parece vincular una parte de la flora rara con el grado de presión antrópica. La misma explicación cabría dar a su disminución con el aumento de la altitud, pero la separación de la rareza vinculada a la actividad humana y la relacionada con rasgos biológicos queda todavía lejos de ser elucidada (Kunin \& Gaston, 1993 y 1997). En cualquier caso, la mayor presencia de plantas raras a baja altitud en los Pirineos contrasta con el patrón encontrado en un sector del Macizo Central en Francia (Lavergne et al., 2005), aunque coincide con el descrito en Gran Bretaña (Hodgson, 1986). El estrecho rango altitudinal que muestran las plantas raras respecto 


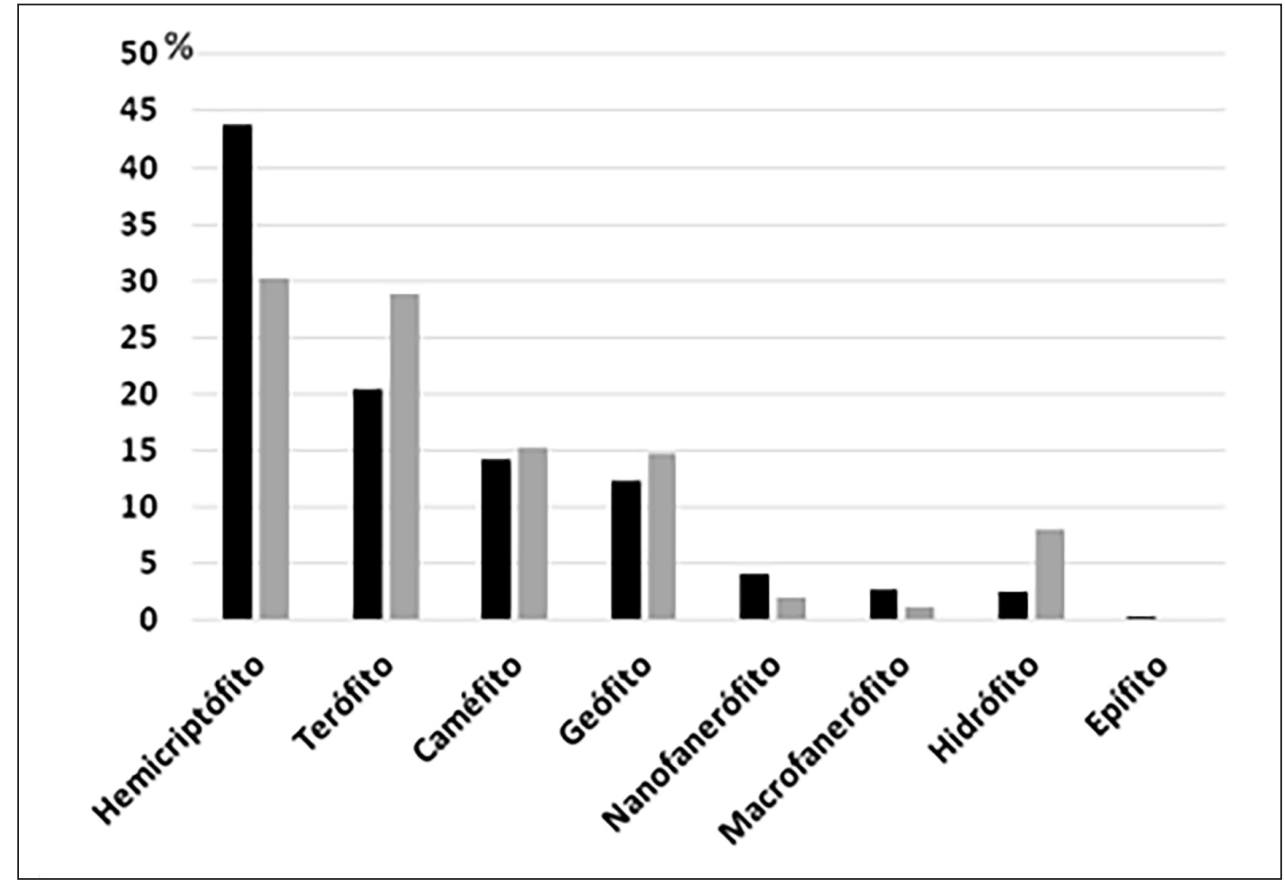

Figura 7: Espectro de formas biológicas de las plantas raras (columnas grises) y del conjunto de la flora de los Pirineos.

Figura 7: Life-form spectrum of the rare plants (grey columns) and the total flora (black columns) of the Pyrenees.

al conjunto de la flora puede indicar en algunos casos una escasa plasticidad frente a las condiciones climáticas (sobre todo de precipitación y temperatura que, en los Pirineos y otras cordilleras alpinas, están estrechamente vinculados a la altitud), aunque el posible sesgo que introduce el escaso número de localidades de las plantas raras aconseja, cuando es posible, la medición de estas variables en áreas de distribución más amplias.

En cuanto a la corología, la sobrerrepresentación de las plantas mediterráneas estaría en parte relacionada con el carácter limítrofe (absoluto o sectorial) que los Pirineos suponen para este grupo (por ej.: Cistus populifolius L. o Helianthemum origanifolium (Lam.) Pers). Sin embargo, en la mayor parte de los casos, se trata de taxones raros en toda su área de distribución (como Limodorum trabutianum Batt.), lo que permite especular que estaríamos frente a un tipo de rareza más relacionado con sus rasgos biológicos o especificidad de hábitat que con los factores ambientales del territorio. Por el contrario, cabe destacar la escasa representación de la flora alpina y boreoalpina entre las plantas raras considerando la minúscula superficie disponible en los niveles altitudinales más elevados. También resulta destacable la notable representación de las plantas de amplia distribución que, entre las raras, incluye una mayoría de taxones que se pueden considerar relictos en cuanto a su distribución (Cosentinia vellea (Aiton) Tod. o Pellaea calomelanos (Sw.) Link) o bien ligados a ambientes como las turberas y otros humedales que resultan muy escasos y sensibles a la perturbación humana (Drosera longifolia L. o Myosurus minimus L.).
Sin embargo, entre las plantas de amplia distribución, algunas son características de entornos muy alterados y su rareza puede ser transitoria (Lemna trisulca L., Spirodela polyrhiza (L.) Schleiden). Por último, la baja representación de la endemoflora entre la flora rara corrobora la escasa relación entre ambos aspectos como ha sido señalado en distintos estudios (Gaston, 1994).

En cuanto a las formas biológicas, la riqueza de los hidrófitos entre las plantas raras parece coincidir con las características del hábitat que ocupan y que acabamos de mencionar. Sin embargo, los terófitos presentan una menor especificidad de hábitats pero también grandes variaciones interanuales en el tamaño de sus poblacionales, lo que unido a su pequeño tamaño supone una complejidad adicional a la interpretación de su rareza, ya que pueden pasar desapercibidos y estar infrarrepresentados en los catálogos. La sub-representación de hemicriptófitos y, sobre todo, de fanerófitos coincide con el patrón encontrado en otros territorios (Lahti et al., 1991), aunque en algunos estudios, la forma biológica no se ha mostrado como una variable diferenciadora entre las plantas raras (Murray et al., 2002).

La abundancia de plantas raras en humedales y suelos salobres parece claramente justificada por las restricciones ambientales de estos hábitats y su reducida superficie en el área de estudio. Sin embargo, las plantas raras no sobresalen en los roquedos, gleras y suelos pedregosos, donde dominan factores abióticos muy severos. Cabe subrayar también la baja representación de la rareza en bosques, matorrales y pastos, ambientes por lo general muy 


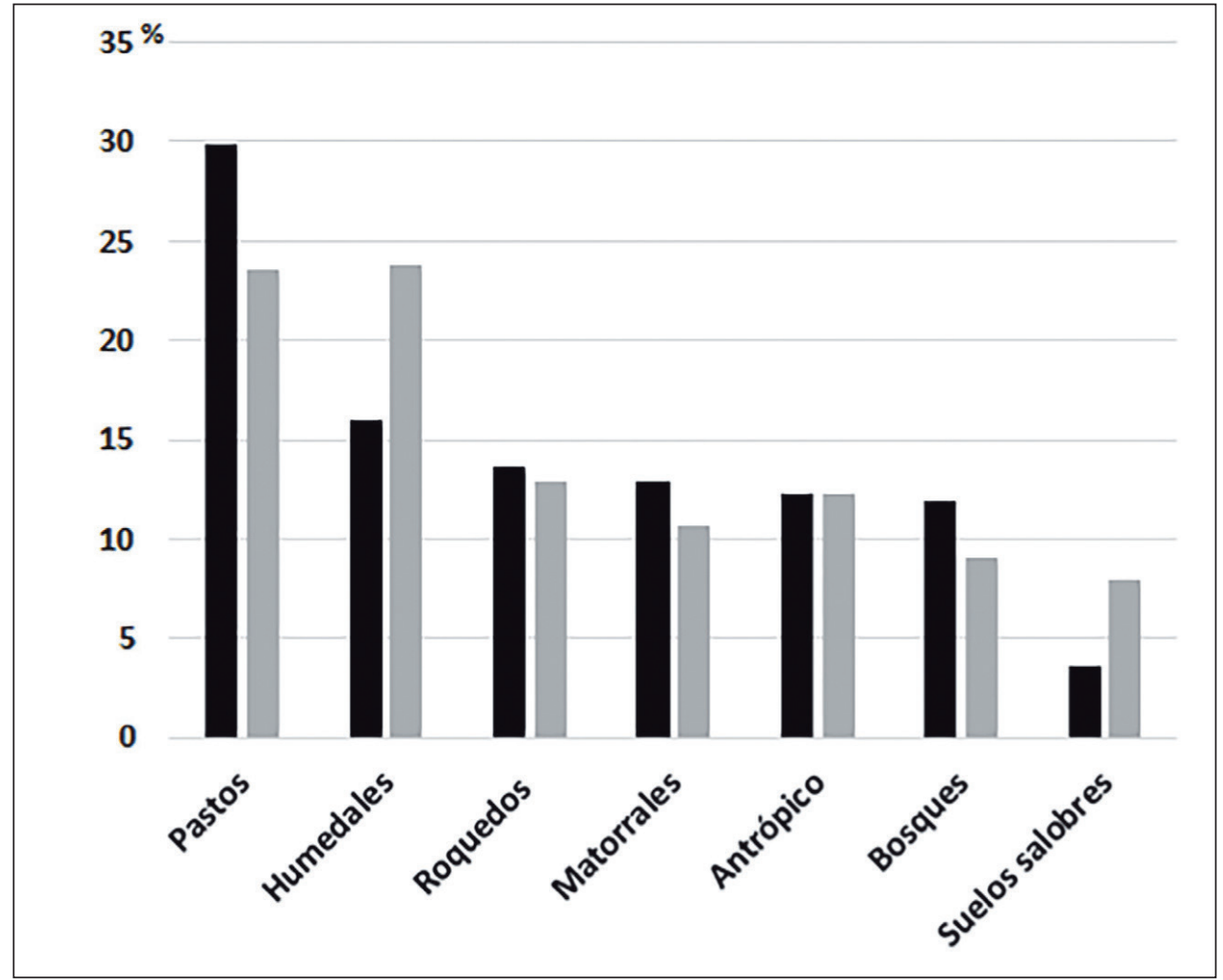

Figura 8: Distribución de las plantas raras (columnas grises) y del conjunto de la flora (columnas negras) en los distintos hábitats de los Pirineos.

Figure 8: Distribution of the rare plants (grey columns) and the total flora (black columns) in the different habitats of the Pyrenees.

intervenidos y perturbados por explotación, fuego y pastoreo ancestral, aunque con marcado abandono en las últimas décadas. Sorprende también la notable presencia de plantas raras en los hábitats más humanizados, aunque esta misma tendencia ha sido encontrada en otros territorios y se ha relacionado con los cambios de uso en las últimas décadas (Hodgson, 1986). En conjunto, alrededor de la mitad de la flora más rara se puede asociar a los hábitats de mayor naturalidad (humedales no eutrofizados y roquedos), es decir con menor intervención humana, mientras que la quinta parte se ubica en los ambientes más intervenidos (pastos degradados, matorrales, bosques muy explotados y terrenos cultivados). Los humedales, por otro lado, constituyen ambientes muy sensibles al cambio climático y a la perturbación humana, por lo que las plantas raras y de alta especificidad que allí viven están sometidas a una alta vulnerabilidad.

Para finalizar, hay que señalar que el análisis de las causas de la rareza requiere un minucioso conocimiento de la historia y los rasgos ecológicos de las especies $(\mathrm{Mu}-$ rray et al., 2002) que, a su vez, resulta esencial en la conservación de la diversidad (Kunin \& Gaston, 1993 y 1997). Aunque la vulnerabilidad de las plantas y el riesgo de extinción no se corresponden en muchos casos con el grado de rareza (Mace \& Kershaw, 1997), las plantas raras constituyen un grupo imprescindible para definir prio- ridades de conservación. Los Pirineos con su gran diversidad geográfica, biológica y larga historia de usos humanos, junto a un grado muy notable de su conocimiento florístico, constituyen un territorio idóneo para la todavía incipiente, y a veces compleja, interpretación de la rareza (Murray et al., 2002) y para aplicar la gestión de la conservación superando los límites de las regiones administrativas que imperan en el presente.

\section{Agradecimientos}

Los datos básicos de este trabajo se han elaborado en parte con la información recogida en el Atlas digital de la flora vascular de los Pirineos, subvencionado por Fondos FEDER de la UE, patrocinado por un Proyecto POCTEFA de la Comunidad de Trabajo de los Pirineos (CTP) y el Observatorio Pirenaico de Cambio Climático (OPCC). Los autores de las fichas de dicho atlas son (por orden alfabético): C. Aedo, I. Aizpuru, J. Ascaso, C. Bergès, M. Domenech, O. Fernández, J.V. Ferrández, X. Font, J. Garmendia, D. Gómez, N. Ibáñez, B. Komac, F. Laigneau, M. Lorda, F. Martínez, J.M. Martínez, J. Molina, N. Montes, J.M. Montserrat, F. Muñoz, C. Navarro, N. Nualart, L. Oreja, J. Pedrol, J. Peralta, D. Pérez, C. Pladevall, J. Puente, A. Pujadas, S. Pyke, J.L. Remón, I. Soriano, J.M. 
Tison, L. Uriarte, P.M. Uribe-Echebarría, A. Valverde. Las instituciones participantes en el proyecto: IHOBE, CSIC (Instituto Pirenaico de Ecología, Jardín Botánico e Institut Botanic), Universitat de Barcelona, Universidad Pública de Navarra, Sociedad de Ciencias Aranzadi, Conservatoire Botanique Mediterranéen, Conservatoire Botanique des Hautes Pyrénées y CENMA de Andorra. En la elaboración de mapas y gráficos tenemos que agradecer la ayuda de Luis Calderón y Paz Errea. El estudio se enmarca dentro del proyecto PERDIVER (Fundación BBVA).

\section{Referencias}

Aizpuru, I., Aseginolaza, C., Uribe-Echebarría, P., Urrutia, P. \& Zorrakin, I., 1999. Claves Ilustradas de la Flora del País Vasco y territorios limitrofes. Servicio central de publicaciones del Gobierno Vasco, 831 pp., Vitoria-Gasteiz

Batianoff, G.N. \& Burgess, R., 1993. Problems in the Documentation of Rare Plants-The Australian Experience. Biodiversity Letters, 1(6): 168-171.

Blackburn, T.M. \& Gaston, K.J., 1997. Who is rare? Artefacts and complexities of rarity determination. In The Biology of Rarity (eds. W.E. Kunin \& K.J. Gaston). Chapman \& Hall. London.

Bolòs, O. de \& Vigo, J., 1984-2001. Flora dels Països Catalans. 4 vols. Ed. Barcino. Barcelona.

Bordère, H., 1866. Découverte du Dioscorea pyrenaica. Bull. Soc. Bot. Fr., 13: 243-244.

Buzas, M.A., Koch, C.F., Culver, S.J. \& Sohl, N.F., 1982. On the Distribution of Species Occurrence. Paleobiology, 8: 143-150.

Carrascal, L.M. \& Palomino, D., 2006. Rareza, estatus de conservación y sus determinantes ecológicos. Revisión de su aplicación a escala regional. Graellsia, 62 (núm. ext.): 523-538.

Carroll R., Augspurger C., Dobson A., Franklin J., Orians G., Reid W. et al., 1996. Strengthening the use of science in achieving the goals of the endangered species act: an assessment by the Ecological Society of America. Ecological Applications, 6: 1-11.

Castroviejo, S. (Coord.). 1986-2015. Flora iberica. Real Jardín Botánico de Madrid-C.S.I.C. Madrid.

Darwin Ch., 1859. On the Origin of species. John Murray, London.

Domínguez Lozano, F., Moreno Saiz, J.C. \& Sainz Ollero, H., 2003. Rarity and threat relationships in the conservation planning of Iberian flora. Biodiversity and Conservation, 12: 1861-1882.

Dupias, G., 1985. Végétation des Pyrénées. Memoire de la Carte de Végétation de la France au 200.000e. Edition du CNRS, 209 pp., Paris.

Dulac, J., 1886. Mélanges botaniques. Plantes nouvelles, critiques, monstrueuses et rares. Vol. 1, 424 pp. Paris.

Drury, W.H., 1974. Rare species. Biological Conservation 6: 162-169. https://doi.org/10.1016/0006-3207(74)90061-5

Fiedler, P.L. \& Ahouse, J.J., 1992. Hierarchies of cause: toward an understanding of rarity in vascular plant species. In: Conservation Biology: the theory and practice of nature conservation, preservation and management (eds. P.L. Fiedler and S.K. Jain). Chapman \& Hall, pp, 23-47, London.

García, M. B., 2003. Demographic viability of a relict population of the critically endangered plant Borderea chouardii. Conservation Biology, 17: 1672-1680.

Gaston, K.J., 1994. Rarity. Chapman and Hall. London.
Gaston, K.J. 1997. What is rarity? In: The Biology of Rarity: Causes and Consequences of Rare-Common Differences. (Ed.: Kunin, W.E. \& Gaston, K.J.) Chapman \& Hall (Population and Community Biology Series 17), 1997. (xiv +280 pages).

Gómez, D., Mateo, G., Mercadal, N, Montserrat, P \& Sesé, J.A: (2005). Atlas digital de la Flora de Aragón. http://floragon. ipe.csic.es

Gómez, D., Lorda, M., Font, X., García, M. B. \& Aizpuru, I., 2016. Rareza en la flora pirenaica. Materiales preliminares para una lista roja. XI Coloquio Internacional de Botánica PirenaicoCantábrica. Señorío de Bertiz. Navarra. (En prensa)

Gómez, D., García, M.B., Font Castell, X., Aizpuru, I., 2017. Distribución espacial y análisis ambiental de la flora vascular de los Pirineos. Pirineos, 172, e028. doi: http://dx.doi. org/10.3989/pirineos.2017.172003

Griggs, R.F., 1940. The ecology of rare plants. Bulletin of the Torrey Botanical Club, 67: 575-594.

He, F. \& Condit, R., 2007. The distribution of species: occupancy, scale and rarity. In: Scaling Biodiversity (ed. David Storch, Pablo A. Marquet and James H. Brown). Published by Cambridge University Press.

Hodgson, J.G., 1986. Commonness and rarity in plants with special reference to the Sheffield flora Part I: The identity, distribution and habitat characteristics of the common and rare species. Biological Conservation 36(3): 199-252. https://doi.org/10.1016/0006-3207(86)90052-2

Humboldt, A. von, 1805. Essai sur la géographie des plantes. Chez Levrault, Schoell et Compagnie, Libraires. XIII Paris.

Keith, D. A., 1998. An evaluation and modification of world conservation union red list criteria for classification of extinction risk in vascular plants. Conservation Biology, 12: 1076-1090

Kunin, W. E. \& Gaston, K. J., 1993. The Biology of Rarity: Patterns, and consequences. TREE, 8(8): 298-301. https://doi. org/10.1016/0169-5347(93)90259-R

Kunin, W. E. \& Gaston, K. J., 1997. On the causes and consecuences of rare-common differences. Extinction risk and rarity on an ecological timescale. In: The Biology of Rarity: Causes and Consequences of Rare-Common Differences. (Ed.: Kunin, W.E. \& Gaston, K.J.) Chapman \& Hall (Population and Community Biology Series 17), 1997. (xiv +280 pages)

Lahti, T., Kemppainen, E., Kurtto, A. \& Uotila, P., 1991. Distribution and biological characteristics of threatened vascular plants in Finland. Biological Conservation 55: 299-314. https://doi.org/10.1016/0006-3207(91)90034-7

Lavergne, S., Thuiller, W., Molina, J. \& Debussche, M., 2005. Environmental and human factors influencing rare plant local occurrence, extinction and persistence: a 115 year study in the Mediterranean region. Journal of Biogeography, 32: 799-811.

Lorda, M., 2001. Flora del Pirineo navarro. Guineana, 7: 557pp., Bilbao.

Lorda, M., Berastegi, A., Gil, T. \& Peralta, J., 2009. Criterios para la priorización de la flora amenazada en Navarra, nuevas perspectivas para la gestión. En: Llamas, F. \& Acedo, C. (coord.) Botánica Pirenaico-Cantábrica en el siglo XXI: 219-243. Universidad de León. Área de Publicaciones. León.

Lorda, M., 2013. Catálogo Florístico de Navarra. Monografías de Botánica Ibérica, 11 Jolube Ed. 281 pp.

Mace, G. M. \& Kershaw, M., 1997. Extinction risk and rarity on an ecological timescale. In: The Biology of Rarity: Causes and Consequences of Rare-Common Differences. (Ed.: Kunin, W.E. \& Gaston, K.J.) Chapman \& Hall (Population and Community Biology Series 17), 1997. (xiv +280 pages).

Montserrat, G. \& Montserrat, J., 1990. Rareza y vulgaridad en la flora de áreas de montaña: el ejemplo de la transición climática atlántico-mediterránea en el Pirineo. In: GarcíaRuiz, J.M. (ed.) Geoecología de las áreas de montaña. Geoforma Ediciones: 145-193 pp., Logroño. 
Murray, B. R., Thrall, P. H., Gill, A. M. \&. Nicotra, A. B., 2002. How plant life-history and ecological traits relate to species rarity and commonness at varying spatial scales. Austral Ecology, 27: 291-310.

Pardo, I., Roquet C., Lavergne J., Olesen J.M. \& García M. B., 2017. Spatial congruence between taxonomic, phylogenetic and functional hotspots: true pattern or methodological artifact?. Diversity and Distributions, 23: 2029-220. https://doi. org/10.1111/ddi.12511

Rabinowitz, D., 1981. Seven forms of rarity. In: Synge, H. The biological aspects of rare plant conservation. John Wiley \& Sons: 205-217 pp., Chichester.

Rodrigues, A. S. L. \& Gaston, K. J., 2002. Rarity and conservation planning across geopolitical units. Conservation Biology 16(3): 674-682.
Schönswetter, P. \& Tribsch, A., 2005. Vicariance and dispersal in the alpine perennial Bupleurum stellatum L. (Apiaceae). Taxon, 54 (3): 725-732

Tison, J. M. \& De Foucault, B., 2014. Flora Gallica-Flore de France. Biotope Éd. Mèze.

Tutin, T. G. et al., (eds.), 1964-1980. Flora Europaea, 5 vols. Cambridge University Press. Cambridge.

Villar, L., Catalán, P., Guzmán, D. \& Goñi, D., 1995. Bases técnicas para la protección de la flora vascular de Navarra. Informe inédito. Gobierno de Navarra-Instituto Pirenaico Ecología (CSIC).

Villar, L., Sesé, J. A. \& Ferrández, J. V., 1997-2001. Atlas de la Flora del Pirineo Aragonés, 2 vols. Instituto de Estudios Altoaragoneses-Consejo de Protección de la Naturaleza. Huesca y Zaragoza. 